Journal of Environmental Assessment Policy and Management

Vol. 18, No. 4 (December 2016) 1650025 (24 pages)

(C) The Author(s)

DOI: $10.1142 / \mathrm{S} 1464333216500253$

\title{
Valuing a Statistical Life Year in Relation to Clean Air
}

\author{
Lars Hein*, Pete Roberts ${ }^{\dagger}$ and Lucia Gonzalez ${ }^{*}$ \\ Department of Environmental Sciences \\ Wageningen University and Research \\ 6700 AA Wageningen, The Netherlands \\ *lars.hein@wur.nl \\ 'pete@peteroberts.onmicrosoft.com \\ tucia.gonzalez@concawe.org
}

Received 22 September 2016

Revised 31 October 2016

Accepted 10 November 2016

Published 28 December 2016

\begin{abstract}
Environmental cost-benefit analysis is increasingly used to support the formulation of European air quality policies. In these analyses, typically around three-quarters of the societal benefits of cleaner air are related to monetised increases in statistical life expectancy. However, the literature presents widely diverging estimates for the value of a statistical life year (a 'VOLY'). This paper presents a review of studies aimed at establishing a VOLY as used in European air quality policies and it examines the factors that cause the variations in VOLY estimates. We discuss the implications of our findings for European air quality policies and also present a novel approach to analyse the VOLY. We have labelled our approach the 'maximum societal revenue VOLY' (MSR-VOLY), and postulate that this approach may be particularly useful in the context of natural capital accounting.
\end{abstract}

Keywords: VOLY; cost-benefit analysis; air pollution; policy; Europe.

\section{Introduction}

European air quality policies have been guided by the 1999 Gothenburg Protocol, which is part of the Convention on Long-Range Transboundary Air Pollution. The

*Corresponding author.

This is an Open Access article published by World Scientific Publishing Company. It is distributed under the terms of the Creative Commons Attribution 4.0 (CC-BY) License. Further distribution of this work is permitted, provided the original work is properly cited. 
protocol includes pollutant mass emission limits for each of the currently 32 signatory parties. In the EU, the European Thematic Strategy on Air Pollution and the National Emission Ceilings (NEC) Directive (2001/81/EC) set parallel targets on air quality in line with the Gothenburg protocol. Central in providing the science behind the 1999 Gothenburg Protocol and NEC Directive was the regional air pollution information and simulation (RAINS) model. This model links economic development and potential abatement measures for various pollutants with the environmental impacts of air pollution. The implementation of the Gothenburg Protocol and NEC Directive has substantially improved European air quality and further improvements are expected through the continuing implementation of the protocol (see, for example, CIAM, 2007, 2011).

In 2005, the EU launched a new set of air quality policies in the form of the Thematic Strategy on Air Pollution (TSAP). The TSAP included several new emission reduction targets (IIASA, 2014). For the TSAP and the recent versions of the NEC directive, cost-benefit analysis (CBA) has been used to compare societal costs and benefits of various air pollution control scenarios (see, for example, IIASA, 2014). Comparison of societal costs and benefits of different policy options can assist policy makers in deciding upon cost-effective ambition levels for environmental policies (Ostro and Chestnut, 1998; Bradley et al., 2008; Desaigues et al., 2011; IIASA, 2013; EEA, 2014). The costs of environmental policies can be relatively straightforward to establish because they require investments in specific, well-understood technologies. These technologies are included in the RAINS model and the 2006 extension of RAINS, the Greenhouse Gas and Air Pollution Interactions and Synergies (GAINS) model (which also includes greenhouse gas emissions). Analysing and valuing the benefits of environmental policies is often, however, more complicated (Nemet et al., 2010; Amann et al., 2011; Bateman et al., 2011; Thompson et al., 2014). Analysing these benefits requires linking policy measures to environmental conditions and subsequently to benefits for people. Benefits of environmental policies may include avoided health impacts or sustaining the supply of ecosystem services.

The most controversial element in CBAs of air quality policies is if and how to value potential reductions or increases in life years of the populations affected by environmental policies. The monetised value of a statistical increase in life expectancy of one year is called the VOLY, i.e. 'value of a statistical life year'. In specific cases, such as analysing the costs of air pollution or the benefits of air pollution control, the value attributed to changes in statistical life expectancy comprises a major share of the total economic value related to health effects. For example, around $70-75 \%$ of the estimated total costs of air pollution in Europe is typically related to the costs attributed to a reduction in life expectancy across the 
population (AEA, 2011; IIASA, 2013). VOLY is therefore a crucial factor driving the monetised benefits of environmental policies.

Over time, input to the societal and policy debates on the monetary benefits of reduced air pollution has been provided by a range of studies on VOLY, both commissioned by the EU Directorate General (DG) for the Environment (see, for example, AEA, 2011; IIASA, 2013, 2014) and studies published in the scientific domain (see, for example Krupnick, 2007; Desaigues et al., 2011). These studies shown a very wide range for VOLY, varying from several thousand euros to well over 100,000 EUR (see, for example, Chilton et al., 2004; Desaigues et al., 2011; Chanel and Luchini, 2014). In addition, there is increasing interest in natural capital accounting, which requires a different approach to monetary valuation compared to CBA (Obst et al., 2016). In natural capital accounting, valuation methods for environmental assets such as clean air are still under development (UN et al., 2014; Obst et al., 2016).

The objective of this paper is to review and analyse the different approaches for estimating VOLY as well as the currently available VOLY estimates related to European air quality. We also explore an additional, complementary metric, the 'maximum societal revenue VOLY' (MSR-VOLY). The MSR-VOLY is not meant to be an alternative for the mean or median VOLY in social cost-benefit analysis. However, it is a potentially relevant indicator to capture the benefits of clean air and the costs of air pollution in a natural capital accounting approach. VOLY is sensitive to the context of the analysis (see, for example, Krupnick, 2007; Hammitt, 2007), and we focus our paper on Europe and in particular on the application of VOLY in the context of the debate on air pollution. The innovations of our paper are to provide an up-to-date review of VOLY in the context of the European air quality debate, and to explore MSR-VOLY as a complementary approach to analyse VOLY. In the discussion of our paper we also reflect upon the potential applicability of MSR-VOLY as a valuation approach for natural capital accounting.

\section{Valuing Health Impacts of Air Pollution}

\section{Measuring health impacts of air pollution}

The Gothenburg Protocol and the TSAP specify emission reductions for a range of pollutants including $\mathrm{SO}_{x}, \mathrm{NO}_{x}, \mathrm{NH}_{3}$, volatile organic compounds (VOCs) (Gothenburg 2012 Amendment), and particulate matter (PM). Health impacts of air pollution are commonly related to the concentration of PM (Ostro and Chestnut, 1998; Pervin et al., 2008). Two sizes of PM are referred to, particles 
with diameters smaller than $10 \mu \mathrm{m}\left(\mathrm{PM}_{10}\right)$ and $2.5 \mu \mathrm{m}\left(\mathrm{PM}_{2.5}\right)$, respectively. The ambient concentrations of these particle types are correlated, $\mathrm{PM}_{10}$ contains $\mathrm{PM}_{2.5}$, but in recent years it has become clear that the smaller fraction poses higher health risk broadly reflecting the ability of these particles to penetrate into the lungs. Note that PM is only defined in terms of the size of the particle and that particles may have very different chemical compositions. A distinction is often made between primary PM, i.e. emitted particles, and secondary PM, which is formed in the lower atmosphere through aggregation of molecules, in particular, sulphur and nitrogen oxides, volatile organic compounds, and ammonia (Mysliwiec and Kleeman, 2002). Primary PM and secondary PM precursors are emitted from a range of sources including emissions from various sectors and natural sources. Both primary and secondary PMs are relevant for the Gothenburg Protocol and the TSAP since these policy documents consider the total amount of particulate matters independent of the origin. Data from epidemiological studies indicates that long-term exposure to $\mathrm{PM}_{2.5}$ can increase human mortality and morbidity risks (Künzli et al., 2000). Reducing $\mathrm{PM}_{2.5}$ concentrations should reduce age-specific mortality risk in a population and consequently result in an increase in the population statistical life expectancy. The parameter that is chosen in policy-support studies to describe this benefit is population life years, which captures both demographic aspects (population size and age distribution) and life expectancy. The effect of $\mathrm{PM}_{2.5}$ is conventionally expressed as the years of life lost (YOLL) associated with the incremental risk. To monetise the health benefit associated with a given reduction in $\mathrm{PM}_{2.5}$, it is therefore necessary to calculate the potential YOLL that would result from this reduction and multiply it by VOLY.

\section{Approaches to measure VOLY}

A range of studies has been devoted to estimate VOLY or, its complementary but different metric, the value of a statistical life (VSL; sometimes also referred to as the value of a prevented fatality, VPF). VSL and VOLY are alternative descriptions of the willingness to pay (WTP) for a specified reduction in a population's hazard function, which reduces mortality risk over a specific period and increases life expectancy (see, for example, Hammitt, 2007). Values for VOLY and VSL are a function of, among other factors, context (specific environmental or health issue at hand) and the income level and age distribution of the affected population (Krupnick, 2007). Two basic approaches are available to analyse VOLY and VSL. The first is to elicit people's WTP by means of a survey. This approach is used to derive VOLY by asking people for their WTP to increase their (statistical) life expectancy by a given period (see, for example, Desaigues et al., 2011), or by 
asking people their WTP to reduce the risk of dying within a specific time period (e.g. 10 years), which can be used to calculate VSL (see, for example, Chanel and Luchini, 2014) from which a VOLY can be derived. Estimating VOLY based on VSL is, however, not straightforward because the risks valued are different for the solicitation of VOLY and VSL, increasing the uncertainty of the VOLY estimate (Leksell and Rabl, 2001). The second approach derives a value for VSL based on decisions people take in their daily lives and that can be related to both a monetary factor (e.g. an expenditure or an increase in salary) and a statistical change in life expectancy (for instance, related to choosing a relatively high-risk job, see Hammitt, 2012). Both estimates of VOLY and of VSL are subject to considerable uncertainty. Estimates for a VOLY range from 6200 EUR (lowest median value for VOLY found by Chilton et al. (2004)) to 150,000 EUR (highest mean value for VOLY found by Chanel and Luchini (2013)). Estimates for the value of VSL range from 0.5 EUR to over 6 million EUR (Hirth et al., 2000; Krupnick et al., 2002).

Hammitt (2007) states that both VSL and VOLY can be appropriate measures to be used in CBA, but that their applicability varies as per the policy question studied. In the case where premature fatality is caused by calamity such as a traffic incident the resulting loss of life expectancy is not known, and the VSL is the more appropriate indicator. On the other hand, in the case of cohort studies which follow over time populations exposed to different levels of air pollution to provide estimates of the survival curve and how it depends on air pollution, the number of life years saved and thereby the VOLY is the more appropriate metric (Hammitt, 2007). In this case, a change in the population survival curve can be the result of extending either the lives of many people for a short time or the lives of fewer people for a longer period rendering VSL less suitable as value indicator (Rabl, 2003). In line with the above, VOLY has been recognised to be the preferred metric to analyse and inform on monetary benefits resulting from improvements in air quality (see Chilton et al., 2004; Desaigues et al., 2011; Luchini and Chanel, 2014).

\section{Challenges in measuring VOLY}

WTP studies give a skewed distribution of results, with a small number of respondents submitting high or very high WTP bids and no respondents bidding lower than zero for an increase in life expectancy. The mean WTP in most surveys considerably exceeds the median WTP. There is still some discussion on if the mean or median value should be used, with recent assessments conducted for DG Environment (see IIASA, 2014) using the median value with the mean value used for sensitivity analysis. An advantage of the median VOLY is that it is less 
sensitive to respondents stating very high WTPs which are not representative or realistic, and which have a large effect on the mean WTP (Desaigues et al., 2011). Calculating VOLY from mean WTP is however better aligned with economic welfare theory, which requires consideration of the actual WTP of each individual in order to calculate the social welfare gained from a certain environmental state (see Freeman, 1993). However, the mean value from individual WTP bids can only be expected to be a robust value if truthful responses are obtained of people's WTP for increased statistical life expectancy or reductions in statistical risks for premature mortality. The number of bids qualified as overly high or low in WTP surveys, and the identification of protest bids, indicates that it cannot be generally assumed that all bids represent truthful responses. In addition, WTP bids are sensitive to the framing of the question, influenced by, for example, information provided prior to the survey, the preceding questions, or the specific WTP options given (see for the latter aspect, for example, Nielsen et al., 2010). In practice, therefore, the studies that analyse VOLY tend to exclude outliers (which are assumed to include zero or unrealistically high bids) from the mean and median VOLY estimates (as in Chilton et al. (2004) and in Desaigues et al. (2011). An issue remains, however, that it is not always easy to identify unrealistic bids, how low or high does a bid have to be before it is considered unrealistic?

Crucial in this regard is the design of the survey instrument used to elicit values in a contingent valuation study (CVM). CVM is an empirical technique for estimating non-market values defined within the standard neo-classical model of valuation (Hanley et al., 2003). When health benefits from reduced pollution are analysed in monetary terms, CVM is used to elicit, by means of surveys, people's WTP to forego adverse health effects including premature mortality (Pervin et al., 2008). When applying contingent valuation surveys to elicit people's WTP for an increase in life expectancy or other good or quality, it is important to understand the various methodological challenges involved (Diamond and Hausman, 1994). Some of the main points are as follows. First, in WTP surveys, there is usually a large variation in responses, and the distribution of responses is necessarily skewed since there is a minimum value that people would respond (a zero WTP for an increase in life expectancy) but no maximum. Second, WTP bids are strongly scale-dependent which presents a problem when scaling results up to a whole life year. Studies show that, proportionately, WTP bids for a short increase in life expectancy (e.g. three months) tend to considerably exceed bids for a longer increase in life expectancy (e.g. six months) when transformed into a WTP for an increase in life expectancy of one year (see Chilton et al., 2004; Desaigues et al., 2011). This may be related to budgetary constraints of respondents (Vatn, 2005) and to difficulties that they may have in linking the bid to the pay-off (Leksell and 
Rabl, 2001) but may also reflect a genuine preference. Third, there may be unrealistically low or high bids including strategic bids or protest bids (of people who believe the question does not make sense, or who believe that it would not be fair for them to pay). All or a proportion of zero or very low bids cast in studies are generally labelled 'protest bids' and omitted. However, it is often very difficult to identify protest and strategic bids (Johannesson et al., 1997). Fourth, the WTP also depends on the perceived future state of health. That is, the willingness to pay to increase life expectancy is less if poor health rather than good health is assumed (Chilton et al., 2004). Fifth, people are found to have difficulty in fully understanding and valuing risk, with WTP for reductions in premature mortality risk leading to very wide ranges (Desaigues et al., 2007). Consequently, the derived bid values for a VOLY are highly sensitive to the framing of the WTP bid, and often show a wide range of WTP bids. This is also reflected in the difference between mean and median values, as we explore in our paper.

\section{Methodology}

\section{Review of VOLY as an indicator for valuing health benefits}

We conducted a review of studies estimating VOLY in the context of European air quality including both mean and median values. We limited ourselves to Europe and air quality because VOLY estimates are strongly influenced by context, both the geographical and cultural contexts and the context in which a WTP for increasing life expectancy is elicited. We searched with Scopus for studies including the words 'VOLY' and 'air pollution' in the abstract and/or keywords, and selected all studies that were conducted in a European country. VOLYs in each of these studies were converted to $€_{2010}$ based on the European (EU-16) consumer price index and where the prevailing exchange rates at the time of the study is relevant. We have included all European studies that we could find in a comprehensive review of the published and grey literature, but acknowledge that we may have missed specific studies and that our review may not be complete.

\section{Defining MSR-VOLY}

Given the limitations in assessing and applying the mean VOLY and the median VOLY as analysed above, we therefore explore an additional approach to calculate VOLY. We have labelled our approach the MSR-VOLY, with MSR standing for the maximum societal revenue. The MSR approach presents a novel way to measure VOLY. It is not intended to replace the mean VOLY as the theoretically correct way of measuring the societal benefits of cleaner air through increases in 
life expectancy. Rather, the MSR-VOLY presents a means for valuing cleaner air that is aligned with a natural capital accounting approach, as discussed below. In addition, we believe MSR-VOLY to be less sensitive to the measurement challenges outlined above, making it potentially a relevant indicator to consider in analysing the benefits of cleaner air.

Basically, the MSR represents the point where the multiplication of a WTP and the number of people expressing at least this WTP is at its maximum. Imagine that society would collect revenue by charging a fee, in return for taking measures reducing air pollution so that the average life expectancy would increase by one year. The fee would only be paid by people whose WTP is equal to or exceeds the fee; hence only people who value an increase in statistical life expectancy enough to justify the air pollution control measures would pay. The maximum societal revenue approach aims to identify the fee that generates the maximum revenue for society, thereby revealing societal demand for clean air. It would be the fee charged by society in the hypothetical case that society would act as a profit maximizing entity able to charge a fee for clean air, under the assumption that this fee would only be paid by people whose WTP equals to or exceeds this fee. Hence, the WTP corresponding to this fee equals the revealed societal demand for an additional life year resulting from cleaner air, and thereby presents an indication of a VOLY appropriate to analyse the monetary value for an increase in life expectancy resulting from cleaner air. We further discuss the methodological implications, as well as how the MSR-VOLY compares to mean and median VOLYs in the context of cost-benefit analysis, in the 'Discussion' section of the paper.

The MSR-VOLY can be calculated using the distribution of existing WTP surveys. Since the original data were not available, we estimated a Weibull distribution for the WTP bids, based on the mean and median values provided by Alberini et al. (2004), Chilton et al. (2004) and Desaigues et al. (2011), using the Gnuplot software package (version 5). The Weibull distribution can be used to characterise a variety of skewed distributions. It has also been used and found as a suitable approach to represent the outcomes of a WTP survey in the context of air pollution, i.e. for representing a WTP survey on air quality in China by Hammitt and Zhou (2006). The Weibull distribution is less restrictive than a normal distribution, and is particularly suited to formalise skewed distributions. The Weibull distribution is represented by Equation (1) below, with $\alpha$ and $\beta$ as scaling parameters. $f(x)$ represents the proportion of people willing to pay an amount $x$ for an increase in statistical life expectancy by one year. The fee corresponding to MSR is calculated by scaling the Weibull distribution over the sample population and by subsequently maximising the societal return obtained by multiplying the WTP with the number of respondents at least willing to pay this fee in return for an 
increase by one year of their statistical life expectancy:

$$
f(x)=\frac{\beta}{\alpha} \cdot\left(\frac{x}{\alpha}\right)^{\beta-1} e^{-\left(\frac{x}{\alpha}\right)^{\beta}} .
$$

The Weibull distribution can be estimated based on the values for mean and median (the relative proportion of the two indicates the skewedness of the sample as captured in the Weibull equations). We estimate the Weibull distribution for a range of WTP surveys related to air quality in Europe. In particular, we analysed the MSR-VOLYs for three WTP surveys conducted by Chilton et al. (2004) and four WTP surveys of Desaigues et al. (2011) (see also Table 1). To analyse MSRVOLY we estimate the Weibull function that best represents these WTP surveys. The two Swiss studies, Soguel and van Griethuysen (2000) and Jeanrenaud and Marti (2007), only present a mean VOLY and without the median VOLY we are not able to estimate the Weibull distribution. For Chilton et al. (2004) we select the (higher) WTP for people being in good health (as presented in Table 1). Desaigues et al. (2011) present two equations that were both used to establish, slightly different, VOLY estimates. Since Desaigues et al. (2011) do not indicate a preference for one equation above the other, we use the average values.

\section{Results}

\section{Review of European VOLY studies}

We found and reviewed seven empirical studies conducted on VOLY in the context of European air quality, see Table 1 (Johannesson and Johansson, 1996; Soguel and van Griethuysen, 2000; Alberini et al., 2004; Chilton et al., 2004; Jeanrenaud and Marti, 2007; Desaigues et al., 2011; Chanel and Luchini, 2014). The surveys are spread over the various European countries (including Czech Republic, Denmark, France, Germany, Hungary, Italy, Poland, Spain, Sweden, Switzerland, and the UK). For six of the studies we were able to retrieve the numbers of respondents: respectively being 566 (Johannesson and Johansson, 1996), 921 (Alberini et al., 2004), 665 (Chilton et al., 2004), 1,463 (Desaigues et al., 2011), 907 (Chanel and Luchini, 2014), and 179 (Jeanrenaud and Marti, 2007). Where available, both the mean and the median VOLYs are presented in Table 1. The difference between the two is reflecting the skewedness of the WTP sample results. The median is typically $40-60 \%$ of the mean VOLY. The exception is the Luchini and Chanel's study that finds a surprisingly similar figure for both mean and median values, suggesting a Gaussian response curve in the case of this study (Chanel and Luchini, 2014). 
L. Hein, P. Roberts \& L. Gonzalez
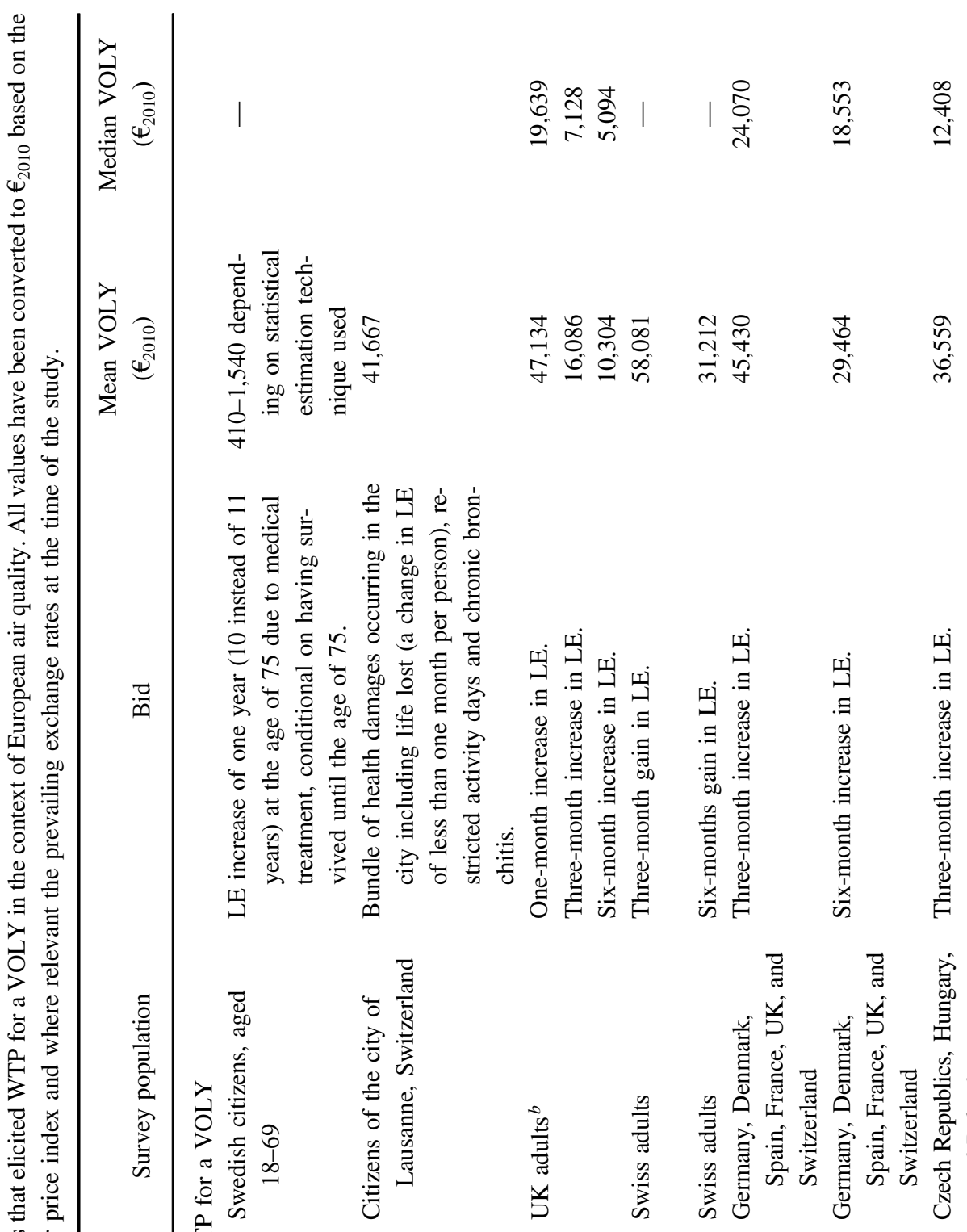

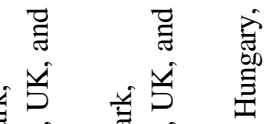

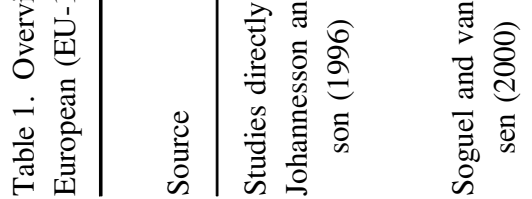

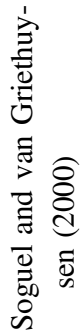

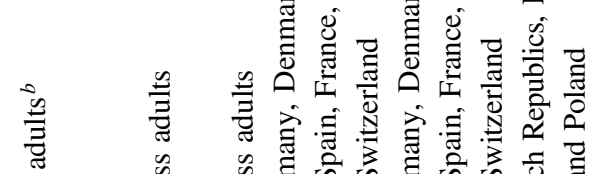

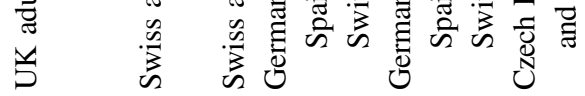


Valuing a Statistical Life Year in Relation to Clean Air

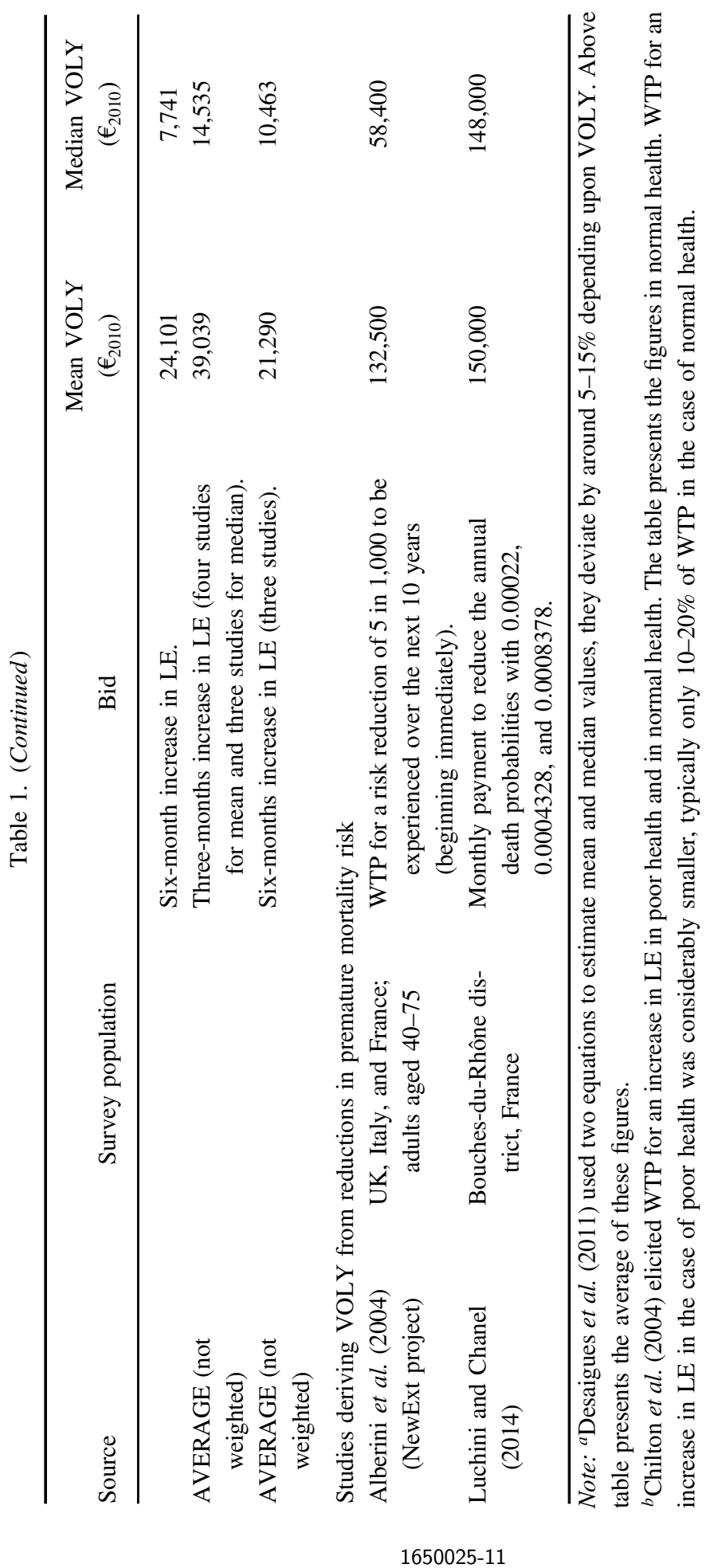


We have grouped the studies in Table 1 into studies that directly elicit bids for increased life-expectancy and those that ask for WTP for a specific premature mortality risk reduction (i.e. the chance of dying in the next 10 years) and then converted it to a VOLY. Specifically, the two studies of Alberini et al. (2004) and Luchini and Channel (2014) based VOLY on the retrieved WTP for a reduction in premature mortality risk whereas the other five studies based VOLY on the WTP for an increase in life expectancy. Table 1 shows that the two studies (Alberini et al., 2004; Luchini and Channel, 2014) that derive a VOLY from a reduced risk for premature mortality find a much higher (a factor 3-4) VOLY compared to studies that directly elicit WTP for increased life expectancy. The difference in VOLYs found in these two groups is substantial, however it is not trivial that there are different outcomes between these two approaches. For instance, Morris and Hammitt (2001) use both approaches to estimate benefits of health care programme and do not find a significant difference between the mean or median VOLYs derived from WTP survey to reduce premature mortality risks and a WTP survey for increased life expectancy. Hence, the marked difference between the two approaches in the European WTP surveys requires further examination, potentially considering the way with which risk was translated into increased life expectancy, something that is complex (Rabl, 2003) (and was also not possible to deduce by us from the two published papers). Table 1 also presents the average mean and median values found in studies directly eliciting WTP. We used an equal weighting for each study, but acknowledge the uncertainty involved given the differences in sample size and potential differences in the quality of the survey instrument.

WTP bids can be expected to strongly vary as a function of the characteristics (age, affluence, environmental awareness, and preferences) of the surveyed population, the design of WTP questionnaire including the specific bid elicited and the framing and phrasing of the bid, and the interpretation of the results. These aspects are reflected in Table 1. The Swiss study found the highest VOLY $(58,000$ EUR) which is likely to be related to Switzerland having one of the highest per capita incomes in Europe. In Eastern and Central European countries a somewhat lower VOLYs were found compared to the Western European countries (Desaigues et al., 2011). Johannesson and Johansson (1996) find a comparatively low VOLY in Sweden. Their result may be explained by the design of the survey which included in particular people of higher age and in poorer health, assuming that the increase in life expectancy was to be enjoyed in a state of poor health. As confirmed in Chilton et al. (2004), WTP for an increase in life expectancy drastically drops when respondents assume that they will be in poor health during the period when they gain in life expectancy. 
Finally, Table 1 points out the importance of WTP bid. VOLY estimates (which are scaled to present the value attributed to a life year) that reflect bids for a six-month or a one-year increase in life expectancy are considerably below estimates derived from a one- or three-month increase. In general, because people's income is limited, the average WTP per month of gain in life expectancy can be expected to decrease with the size of the gain. It is unclear if there could, in addition, be a reduction in the marginal utility attributed to additional increases in life expectancy, as generally assumed for consumed goods. There is no published consensus on the appropriate length of the increase in life expectancy in the context of European air quality policies. Intuitively, it could be assumed that the length of the increase in life expectancy to be valued with a WTP study should be in line with the approximate effects of air pollution, which is in the order of a reduction of life expectancy with 3-6 months in most European countries (see, for example, Künzli et al., 2000; Brunekreef and Holgate, 2002).

\section{The MSR approach to measure VOLY}

The MSR-VOLY represents a novel way to analyse VOLY. It is potentially aligned with a natural capital accounting approach to valuation (Obst et al., 2016), see the 'Discussion' section. It also has the potential advantage that both high and low outliers do not significantly affect VOLY, and that there is no need for arbitrary selection of outliers on either the lower or the higher boundary of the range of WTP estimates.

The Weibull equations calculated for the European WTP studies and the VOLY representing MSR are presented in Table 2. We have also added the median and mean values calculated based on our Weibull equation for all samples, in order to indicate the accuracy of the Weibull curve in representing the datasets. Since the Weibull equations were fitted through the mean and median, there should be a good correspondence with the mean and median VOLYs presented in Tables 1 and 2 (the average difference between our fit and the actual mean and median VOLYs of the different surveys is $0.6 \%$ ).

The MSR-VOLY is, in all surveys, somewhat lower than the median VOLY, ranging from $10 \%$ lower in the case of Desaigues et al. (2011) six months (New Member States), to $44 \%$ lower in the case of Desaigues et al. (2011) three months (EU-16 + Switzerland). The reason for MSR-VOLY being lower than the mean value is that - as does the median - the MSR-VOLY provides relatively little weight to high bids (most important for MSR-VOLY are the bids close to MSRVOLY). The MSR-VOLY is also generally lower than the median because of 


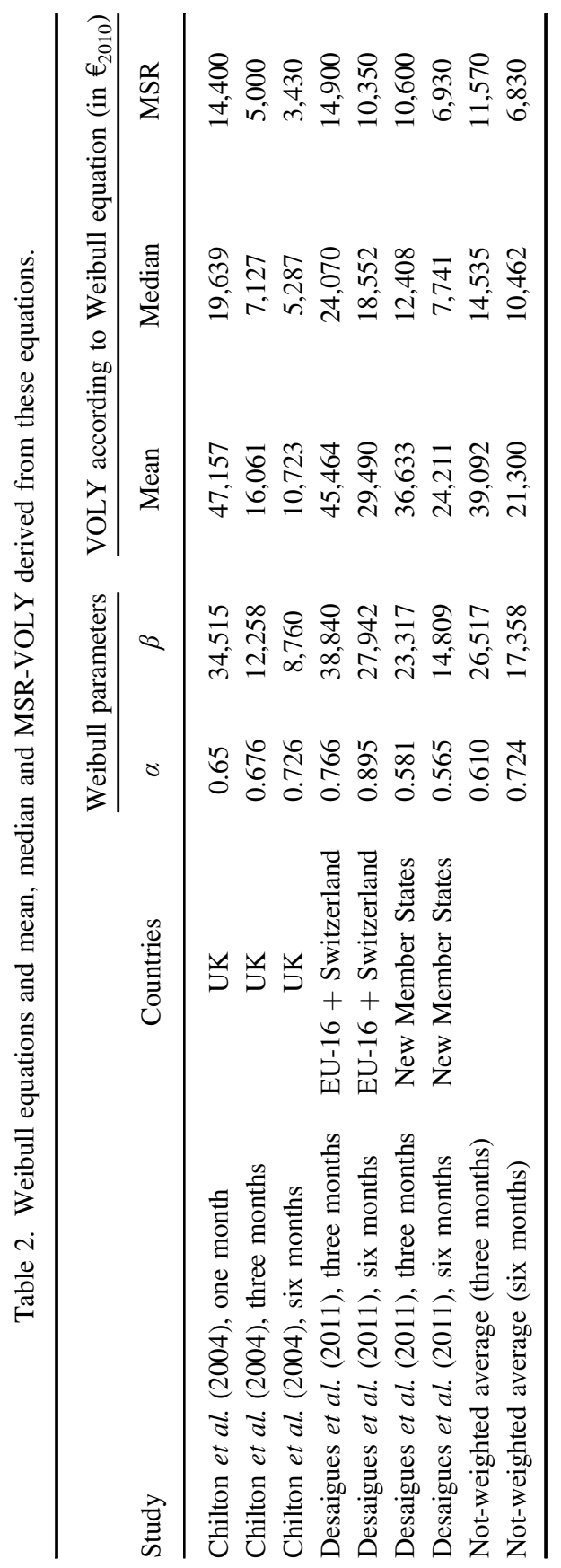


the skewedness of the distribution, there will generally be more people bidding a specific WTP just below compared to just above the median VOLY.

The MSR-VOLY represents the (hypothetical) maximum producer surplus society could obtain in case society would be able to offer life years, at a price, to people in that society. Clearly, this is a theoretical construct, with clean air being a public good that is not tradeable either for practical or moral reasons in a market. In other words, MSR-VOLY represents the value of a life year in case a valuation approach based on exchange values (as in the case of natural capital accounting) rather than welfare-based valuation principles is pursued. The MSR-VOLY approach is, in this sense, comparable to the simulated exchange value approach proposed by Campos and Caparrós (2011) for ecosystem services, as discussed below.

The relation between the median, mean, and MSR-VOLY is explained graphically in Fig. 1. The societal revenue curve (right-hand vertical axis) is obtained by multiplying the WTP curve (left-hand vertical axis) with the number of people willing to pay the stated amount. The maximum of the societal revenue curve presents the MSR-VOLY (indicated). The median value can be derived from the WTP curve. At the point of the median WTP (w2), the number of people stating a lower WTP equals the number of people stating a higher WTP. This is visualised by colouring the area under the WTP curve left of the median value.

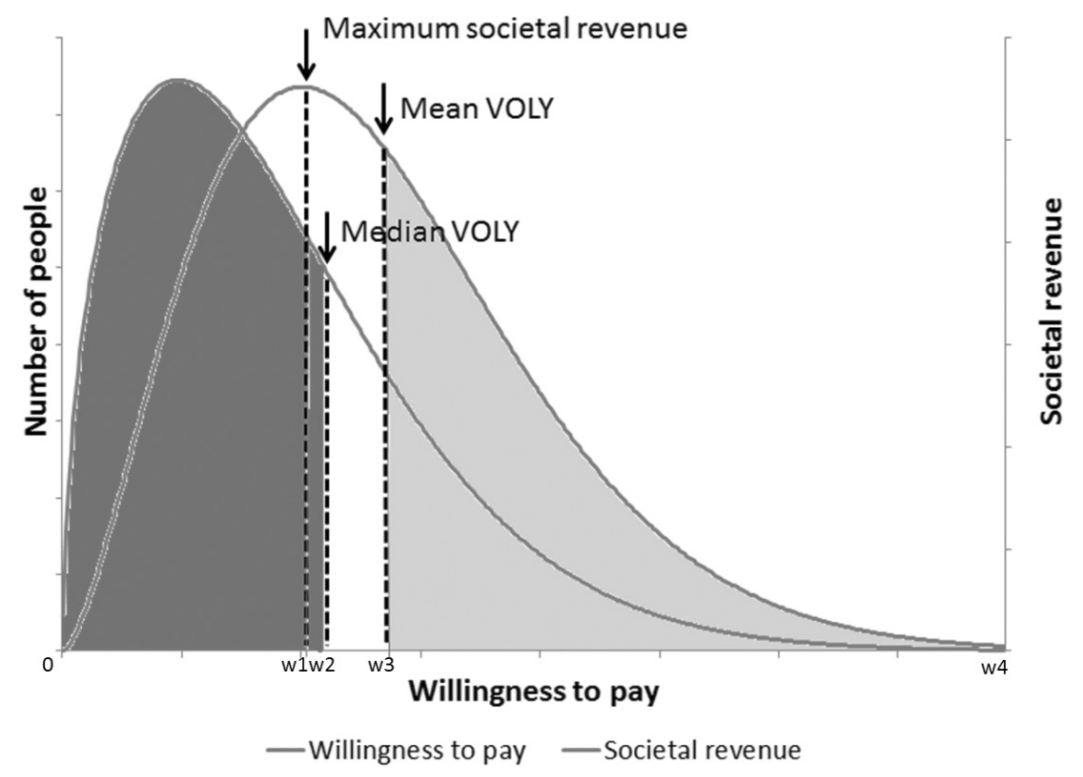

Fig. 1. Median, mean and MSR-VOLYs compared. 
The mean value can also be retrieved from the figure, by looking at the societal revenue curve. At the mean VOLY (indicated by w3), the willingness to pay per person multiplied with the number of persons willing to pay this amount, i.e. the societal revenue, to the left of w3, equals the societal revenue to the right of w3. This is visualised in the figure by colouring the area under the societal revenue curve to the right of the mean value (w3).

\section{Discussion}

\section{Selecting a VOLY for cost-benefit analysis}

There is a high variation in the VOLY estimates found in European studies analysing the WTP for better air quality. The CBA analyst is faced with two fundamental choices: (i) if VOLY should be estimated using a risk-based approach or based directly on WTP for a statistical increase in life expectancy; and (ii) if mean or median VOLY should be used in CBA.

In general, the literature points to three reasons why a VOLY, in the context of analysing the benefits of reduced air pollution, should preferably be based on the WTP for an increase in life expectancy (see, for example, Rabl, 2003; Brunekreef et al., 2007; Nielsen et al., 2010; Desaigues et al., 2011). First, respondents may relate a risk reduction to a change in the occurrence of an accidental death, whereas air pollution shifts the timing of death without a significant change in the nature of the death. Second, people have major difficulties in understanding risk and in attributing a monetary value to a reduction in premature mortality risk; responses may be strongly influenced by the framing of the questions (Klose, 1999; Krupnick et al., 2002; Whynes et al., 2004). Third, analysing VOLY based on a risk-based approach requires an additional analytical step to translate risk reduction to life expectancy as a function of the population's age distribution, health, and changes in health condition and age distribution as a function of (changes in) pollution exposure. For instance, implicit in the valuation of the NewExt study is the assumption that the valued risk reduction (of death occurring in the following 10 years) equates to an overall 37-day increase in life expectancy (Rabl, 2003; AEAT, 2005). This step adds additional complexity and uncertainty to the analysis.

Second, there is the issue of using the mean or the median value. In case the WTP bids can be assumed to reflect truthful and accurate responses, the mean value is to be preferred given that a high WTP of specific individuals in society for clean air needs to be considered in the societal, aggregated WTP for clean air. However, the problem is that individuals have major difficulties responding to 
questions related to premature mortality and life expectancy. Several studies have pointed to the challenges involved in ensuring that respondents to WTP surveys properly understand the proposition they are supposed to bid for, and in particular the challenges related to understanding risks (see Diamond and Hausman, 1994; Klose, 1999; Whynes et al., 2004). Because zero bids are routinely excluded as protest bids, and because there can be no negative bids (in principle), using mean values leads to a bias given that overly high bids may not be corrected (and that it is difficult to identify overestimates). The pragmatic option selected in European CBAs therefore has been to use the median (lower) VOLY, with the mean value used for sensitivity analysis (see IIASA, 2013, 2014). However, this does not solve the problem of subjectivity in selecting protest bids and the problem that uncertainty in the WTP bids means that the mean or median value itself may be over- or under-estimated (i.e. the actual median and mean societal VOLYs may be lower or higher than the median and mean VOLYs retrieved from WTP surveys).

Based on the review presented above, the values found in the literature can be compared with the actual values used in cost-benefit studies conducted by or for European institutions (see AEA, 2011; IIASA, 2013, 2014; EEA, 2011, 2014). These studies all use a VOLY of around $€ 58,000$ based on the median value of the NewExt research project (Alberini et al., 2004). Note that the values in these various studies differ somewhat because they are adjusted for inflation in order to express the VOLY in $€_{2005}$ or $€_{2010}$ depending upon the study. The selection of only Alberini et al. (2004) to inform EU policy-making seems not representative given the differences in values found by the other European studies; Alberini et al. (2004) provide a value that is higher than most other studies (see Table 1).

More work, therefore, is needed in terms of developing mean and median values that are more representative of the values found in the various European studies presented in Table 1, and to design an approach for sensitivity analysis that reflects uncertainty levels in both mean and median values. In addition, potential additional indicators for VOLY should be explored, such as the MSR-VOLY, which is explained in the next subsection. To enhance the understanding of how risk-based surveys relate to life expectancy-based surveys, it would be useful to examine to what degree the respondents are able to adequately comprehend the value reductions in premature mortality risk. To test the performance of the survey instrument, Hammitt and Liu (2004) propose that the sensitivity of estimated WTP to magnitude of risk reduction can be used as a diagnostic test assuming that, under the conventional economic theory, WTP for a small reduction in premature mortality risk is nearly linear in the magnitude of the risk reduction. If original data are still available, the test could potentially also be applied to previous surveys (Hammitt and Liu, 2004). 


\section{On MSR as a complementary metric in CBA and natural capital accounting}

The VOLY represents the mean or median value where people are indifferent between the (theoretical) wealth transfer involved and a life year. In general, the economic value of a public good equals the sum of individuals' WTPs (i.e. the mean VOLY times the number of people). As mentioned earlier, median VOLY has been proposed in consideration of the bias obtained in WTP surveys (where people do not have to actually pay, and where there is a lower bound on values that are excluded in the calculation of the mean but where it is difficult to analyse the upper bound of these values). In proposing an additional index (i.e. the MSR), it is essential to examine and report its properties and how they compare with the currently used values.

Our new method, MSR, presents a metric that is somewhat different from the mean VOLY. The MSR corresponds to the maximum revenue society would obtain if they would be able to levy a fee that would only be paid by people whose WTP for an increased life expectancy of one year due to cleaner air is equal to or larger than this fee. In the terminology of natural capital accounting following the UN System of Environmental Economic Accounting (UN-SEEA) (UN et al., 2014), the MSR is akin to the simulated exchange value of a potential increase in life expectancy from reduced air pollution. The term simulated exchange value was coined by Campos and Caparrós (2011), as further discussed below. It reflects the value the VOLY would have if there would be a market with informed buyers and a profit maximising seller of clean air, in case the seller would not need to pay for providing this clean air (if the seller would have to pay for each 'unit' of clean air provided the optimal amount to be sold would be lower than MSRVOLY, depending upon the shape of the supply curve). We do not further consider the costs of providing clean air in this paper, even though this would be possible using the cost curves for clean air measures included in the IIASA's GAINS model. Obviously, the MSR is a theoretical construct only, we do not suggest the MSR to be a concept that should be used to design actual payment mechanisms. We also recognise that societal cost-benefit analysis generally involves using a welfarebased approach given that the full range of welfare effects including producer surplus and consumer surplus needs to be considered in policy-making (see Freeman, 1993).

The main use of MSR, therefore, is to provide an additional indicator for VOLY reflecting exchange values (see Obst et al., 2016) rather than a welfarebased valuation approach. In addition to being potentially relevant for natural capital accounting, an advantage of MSR is that it does not require subjective 
assumptions on when to classify a WTP bid as unrealistically low or high; the MSR is only sensitive to the WTP distribution of respondents close to the median. The MSR-VOLY is somewhat lower than the median VOLY, and can therefore be seen as a relatively conservative indicator in the face of valuation estimates prone to considerable uncertainty. The MSR is relevant as an additional indicator for sensitivity analysis in CBA, given that it is less sensitive to assumptions on outliers. It needs to be considered in this context that MSR-VOLY reflects not the full welfare value but a value based on exchange values. Basically a valuation based on exchange values aims to estimate (market and non-market) values related to consumption and production, rather than the overall welfare effect. In particular, consumer surplus is excluded from a valuation approach based on exchange values (see, for example, Obst et al., 2016) for more details on the implications of the differences between the two approaches). See also Fig. 1 for a presentation of the difference between MSR, median VOLY, and the mean VOLY.

Given that MSR provides a simulated exchange value, the MSR indicator may also be useful for the emerging field of ecosystem accounting, a recent addition to UN-SEEA (UN et al., 2014). This approach uses the valuation approach of the national accounts (UN et al., 2009) to analyse and record environmental assets providing ecosystem services (UN et al., 2014). A main challenge in ecosystem accounting is finding value indicators for ecosystem services that are not traded in a market and for which no alternative means are available to estimate the potential exchange value for these services (see Edens and Hein, 2013). Campos and Caparrós (2011) developed the simulated exchange value approach to estimate a demand curve and a supply curve for a non-market ecosystem service, tourism, in a forest ecosystem. They analysed the price that would be charged by a profitmaximizing resource manager and subsequently analysed the hypothetical revenue associated with this transaction. This is unlike the MSR-VOLY approach, which, from a theoretical perspective, can be linked to the price society would charge for clean air if it would act as a profit-maximizing resource manager. Further research is needed to examine if and how the MSR approach can be used to value environmental assets in ecosystem accounting.

\section{Conclusions}

VOLY is one of the most important factors that determine the cost-benefit ratio of European air quality policy options. This paper compares the outcomes of seven studies quantifying VOLY in the context of European air quality and presents MSR-VOLY as an additional metric that, pending further testing, may be 
applicable in natural capital accounting where a specific valuation approach is needed to monetise ecosystem assets (Obst et al., 2016).

Our review shows that there are two main approaches that are currently used to analyse a VOLY. Both have been applied in the context of air pollution control in Europe. The first approach bases VOLY values on surveys eliciting WTP for increases in statistical life expectancy (Johannesson and Johansson, 1996; Soguel and van Griethuysen, 2000; Chilton et al., 2004; Jeanrenaud and Marti, 2007; Desaigues et al., 2011). The second approach involves eliciting WTP bids for a decrease in premature mortality risk due to cleaner air (Alberini et al., 2004; Luchini and Channel, 2014). The average mean VOLY values found in the first group of studies are only about one-quarter of the average values found in the second group. The first approach is preferable in CBA (Rabl, 2003; Brunekreef et al., 2007; Hammitt, 2007; Desaigues et al., 2011). We found that many European policy-support studies analysing the benefits of cleaner air use only one of these studies for deriving a VOLY. This study uses a risk-based approach (see Alberini et al., 2004). The use of only one, risk-based, study as a basis for European cost-benefit analyses may lead to overestimation of the benefits of air pollution control measures, and a bias in the cost-benefit ratio of the policy scenarios examined in these studies.

We propose an additional indicator to estimate VOLY, based on MSR that would be obtained if people would have to pay a fee in return for obtaining cleaner air and which would only be paid if their WTP equals or exceeds this amount. The MSR-VOLY is an indicator that reflects the societal demand for cleaner air, and has the advantage that it is not sensitive to low and high outliers in the WTP curve. The MSR-VOLY does not require the selection of thresholds above or below which WTP estimates for gains in life expectancy are classified as outliers. We postulate that the MSR value is aligned with a natural capital accounting approach to valuation. The MSR-VOLY presents an estimate at the lower range of value estimates for clean air in Europe, with particular relevance in a natural capital accounting context.

\section{References}

AEA (2011). Cost-benefit analysis for the revision of the National Emission Ceilings

Directive: Policy options for revisions of the Gothenburg Protocol to the UNECE Convention on Long-range Transboundary Air Pollution. No. ED47788, Report to European Commission, AEA, UK. 
AEAT (2005). Methodology for the Cost-Benefit Analysis for CAFE: Health Impact Assessment, Vol. 2. Oxon: AEA Technology Environment.

Alberini, A, A Hunt and A Markandya (2004). Willingness to pay to reduce mortality risks: Evidence from a three-country contingent valuation study. Report Nota di Lavorro 111.2004, Fondazione Eni Enrico Mattei Note di Lavorro Series, September 2004.

Amann, M, I Bertoka, J Borken-Kleefeld, J Cofala, C Heyes, L Höglund-Isaksson, Z Klimont, B Nguyen and M Posch (2011). Cost-effective control of air quality and greenhouse gases in Europe: Modeling and policy applications. Environmental Modelling and Software, 26(12), 1489-1501.

Bateman, IJ, GM Mace, C Fezzi, G Atkinson and K Turner (2011). Economic analysis for ecosystem service assessments. Environmental Resource Economics, 48, 177-218.

Bradley, CJ, KR Yabroff, B Dahman, EJ Feuer, A Mariotto and ML Brown (2008). Productivity costs of cancer mortality in the United States: 2000-2020. Journal of the National Cancer Institute, 100(24), 1763-1770.

Brunekreef, B and ST Holgate (2002). Air pollution and health. The Lancet, 360, 1233-1242.

Brunekreef, B, BG Miller and JF Hurley (2007). The brave new world of lives sacrificed and saved, deaths attributed and avoided. Epidemiology, 18(6), 785-788.

Chanel O and S Luchini (2014). Monetary values for risk of death from air pollution exposure: A context-dependent scenario with a control for intra-familial altruism. Journal of Environment Economics and Policy, 3(1), 67-91.

Chilton, S, J Covey, M Jones-Lee, G Loomes and H Metcalf (2004). Valuation of health benefits associated with reductions in air pollution. Final Report PB 9413, Defra Publications, London, UK.

Campos, P and A Caparrós (2011). RECAMAN PROJECT: Mediterranean Monte ecosystems total income green accounting. Presentation at the Expert Meeting on Ecosystem Accounting, May 2011, European Environment Agency, Copenhagen.

CIAM (2007). Review of the Gothenburg Protocol. CIAM Report 1/2007, Report of the Task Force on Integrated Assessment Modelling and the Centre for Integrated Assessment Modelling. CIAM, IIASA, Laxenburg, Austria, 2007.

CIAM (2011). Cost-effective emission reductions to improve air quality in Europe in 2020. Background paper for the 48th Session of the Working Group on Strategies and Review, Version 2.1, Centre for Integrated Assessment Modelling (CIAM) and International Institute for Applied Systems Analysis (IIASA). Laxenburg, Austria, 2011.

Desaigues, B et al. (2011). Economic valuation of air pollution mortality: A 9-country contingent valuation survey of value of a life year (VOLY). Ecological Indicators, 11, 902-910.

Desaigues, B, A Rabl, D Ami, BM Kene, S Masson, M-A Salomon and L Santoni (2007). Monetary value of a life expectancy gain due to reduced air pollution: Lessons from a contingent valuation in France. Revue d'économie politique, 117, 675-698. 
Diamond, PA and JA Hausman (1994). Contingent valuation: Is some number better than no number? Journal of Economic Perspectives, 8, 45-66.

Edens B and L Hein (2013). Towards a consistent approach for ecosystem accounting. Ecological Economics, 90, 41-52.

EEA (2011). Revealing the costs of air pollution from industrial facilities in Europe. Technical Report No. 15/2011, European Environment Agency, Copenhagen.

EEA (2014). Costs of air pollution from European industrial facilities 2008-2012: An updated assessment. Technical Report No. 20/2014, European Environment Agency, Copenhagen.

Freeman, AM (1993). The Measurement of Environmental and Resource Values Resources for the Future. Washington, DC: Island Press.

Hammitt, JK (2007). Valuing changes in mortality risk: Lives saved versus life years saved. Review of Environmental Economics and Policy, 1(2), 228-240.

Hammitt JK and JT Liu (2004). Effects of disease type and latency on the value of mortality risk. Journal of Risk and Uncertainty, 28(1), 73-95.

Hammitt, JK and Y Zhou (2006). The economic value of air-pollution-related health risks in China: A contingent valuation study. Environmental and Resource Economics, 33, 399-423.

Hanley, N, M Ryan and R Wright (2003). Estimating the monetary value of health care: Lessons from environmental economics. Health Economics, 12(1), 3-16.

Hirth, RA, ME Chernew, E Miller, M Fendrick and WG Weissert (2000). Willingness to pay for a quality-adjusted life year: In search of a standard. Medical Decision Making, 20(3), 332-342.

IIASA (2013). Policy scenarios for the revision of the thematic strategy on air pollution. TSAP Report No. 10, Version 1.0, Service Contract on Monitoring and Assessment of Sectorial Implementation Actions (ENV.C.3/SER/2011/0009), International Institute for Applied Systems Analysis (IIASA), March 2013.

IIASA (2014). The final policy scenarios of the EU Clean Air Policy package. TSAP Report No. 11, Version 1.1a (revised 28 February 2014), International Institute for Applied Systems Analysis (IIASA).

Jeanrenaud, C and J Marti (2007). The cost of reduced life expectancy due to air pollution: Assessing the value of a life year (VOLY) using contingent valuation. In Proc. iHEA 2007 6th World Congr.: Explorations in Health Economics Paper. Philadelphia: iHEA.

Johannesson, M and P-O Johansson (1996). To be, or not to be, that is the question: An empirical study of the WTP for an increased life expectancy at an advanced age. Journal of Risk and Uncertainty, 13, 163-174.

Johannesson, M, P-O Johansson and K-G Lofgren (1997). On the value of changes in life expectancy: Blips versus parametric changes. Journal of Risk and Uncertainty, 15, 221-239.

Klose, T (1999). The contingent valuation method in health care. Health Policy, 47, 97-123. 
Krupnick, A (2007). Mortality-risk valuation and age: Stated preference evidence. Review of Environmental Economics and Policy, 1(2), 261-282.

Krupnick, A, A Alberini, M Cropper, N Simon, B O'Brien, R Goeree and M Heintzelman (2002). Age, health, and the willingness to pay for mortality risk reductions: A contingent valuation survey of Ontario residents. Journal of Risk and Uncertainty, 24(2), 161-186.

Künzli, N, R Kaiser, S Medina, M Studnicka, O Chanel, M Herry, F Horak, V Puybonnieux-Texier, P Quenel, J Schneider, R Seethaler, J-C Vergnaud and H Sommer (2000). Public-health impact of outdoor and traffic-related air pollution: A European assessment. Lancet, 356, 795-801.

Leksell, I and A Rabl (2001). Pollution and mortality: Quantification and valuation of years of life lost. Risk Analysis, 21(5), 843-857.

Mysliwiec, MJ and MJ Kleeman (2002). Source apportionment of secondary airborne particulate matter in a polluted atmosphere. Environmental Science and Technology, 36 (24), 5376-5384.

Morris, J and JK Hammitt (2001). Using life expectancy to communicate benefits of health care programs in contingent valuation studies. Medical Decision Making, 21(6), 468-478.

Nemet, GF, T Holloway and P Meier (2010). Implications of incorporating air-quality cobenefits into climate change policymaking. Environmental Research Letters, 5(1), 014007.

Nielsen, JS, S Chilton, M Jones-Lee and H Metcalf (2010). How would you like your gain in life expectancy to be provided? An experimental approach. Journal of Risk and Uncertainty, 41(3), 195-212.

Obst, C, L Hein and B Edens (2016). National accounting and the valuation of ecosystem assets and their services. Environmental and Resource Economics, 64(1), 1-23.

Ostro, B and L Chestnut (1998). Assessing the health benefits of reducing particulate matter air pollution in the USA. Environmental Research, 76, 94-106.

Pervin, T, UG Gerdtham and CH Lyttkens (2008). Societal costs of air pollution-related health hazards: A review of methods and results. Cost Effectiveness and Resource Allocation, 6, 19.

Rabl, A (2003). Interpretation of air pollution mortality: Number of deaths or years of life lost? Journal of the Air and Waste Management Association, 53(1), 41-50.

Soguel, N and P van Griethuysen (2000). Evaluation contingente, qualité de l'air et santé: uneétudeen milieu urbain. Cahiers de l'IDHEAP No. 185, IDHEAP, Chavannes-prèsRenens, Switzerland.

Thompson, TM, S Rausch, RK Saari and NE Selin (2014). A systems approach to evaluating the air quality co-benefits of US carbon policies. Nature Climate Change, 4, 917-923.

UN et al. (2014). System of Environmental-Economic Accounting 2012: Experimental Ecosystem Accounting. USA, New York: United Nations. 
United Nations, European Commission, International Monetary Fund, Organisation for Economic Co-operation and Development (2009) System of National Accounts 2008. UN, New York, 2009.

Vatn, A (2005). Institutions and the Environment. UK, Cheltenham: Edward Elgar.

Whynes, DK, J Wolstenholme and E Frew (2004). Evidence of range bias in contingent valuation payment scales. Health Economics, 13, 183-190. 